\title{
Blood flow simulations in patient-specific coronary bypass grafts
}

\author{
E. S. Bernad ${ }^{1}$, M. Craina ${ }^{1}$ \& S. I. Bernad ${ }^{2}$ \\ ${ }^{1}$ University of Medicine and Pharmacy Timisoara, Timisoara, Romania \\ ${ }^{2}$ Romanian Academy - Timisoara Branch, Timisoara, Romania
}

\begin{abstract}
The purpose of this paper was to investigate the hemodynamic parameters associated with the blood flow in failed coronary venous bypass grafts using a computational fluid dynamics (CFD) technique. A narrowed section creates bypass graft constriction and disturbed flow. This change of the flow direction alters significantly the local hemodynamic parameters. In this paper, a patient specific three-dimensional bypass model is used to investigate blood flow in narrowed venous bypass grafts so as to understand the detailed flow physics of the transition to turbulence downstream of the graft narrowed section. Results of the simulation show that successive curvature and sudden narrowing of the bypass graft induce disturbance of the flow profile that produces high local wall shear stress (WSS) and large recirculation regions. Both these parameters create favorable conditions for the initiation of graft failure.
\end{abstract}

Keywords: coronary artery diseases, bypass graft, numerical simulation.

\section{Introduction}

Coronary artery diseases are caused by atherosclerosis. Atherosclerosis involves the development of plaque in the inner sides of large and medium size arteries. The purpose of vascular surgery is to restore blood flow to organs and tissues. The ideal is to restore sufficient blood flow appropriate to the physiologic flow conditions. Usually in coronary arteries, assessment of the hemodynamic flow is performed with intravascular Doppler ultrasound [1-4]. An alternative for this procedure is the computational fluid dynamics (CFD) method used to simulate the blood flow in coronary arteries using geometric models from CT (computed tomography) datasets [5]. 


\subsection{Graft failure}

Bypass failure in the first 10 days after a procedure is commonly associated with technical problems in the course of the operation [6,7]. Graft failure in the period of the first 2 years after the operation is mostly due to the evolving intimal hyperplasia and the progression of atherosclerotic lesions in the native inflow and outflow vessels $[4,8]$. For investigations of bypass failure, CFD can offer effective tools to improve the success rates of the revascularization procedure.

\section{Materials and method}

Spiral CT (computed tomography), Somatom Sensation 64 Scanner (Siemens Medical Systems, Erlangen, Germany) was performed 7 days following the cardiac intervention (bypass surgery). The coronary angiography (CA) and the spiral CT investigation detected a multiple sever stenosis on the right coronary artery (RCA) (Table 1). The clinical solutions for this patient are the venous bypass graft for the RCA (Figures 1 and 2). Data corresponding to the investigated patient is presented in Table 2 .

Table 1: RCA stenosis severity.

\begin{tabular}{cccc}
\hline Stenosis & $\begin{array}{c}\text { Reference } \\
\text { diameter }[\mathrm{mm}]\end{array}$ & $\begin{array}{c}\text { Stenosis minimal } \\
\text { diameter }[\mathrm{mm}]\end{array}$ & $\begin{array}{c}\text { Diameter reduction } \\
\text { percentage [\%] }\end{array}$ \\
\hline ST1 & 3.8 & 1.76 & 54 \\
ST2 & 3.6 & 2.6 & 28 \\
ST3 & 3.6 & 1.68 & 53 \\
\hline
\end{tabular}

Table 2: $\quad$ Investigated patient characteristics.

\begin{tabular}{|c|c|}
\hline Variables & Value \\
\hline Mean age & 54 \\
\hline Gender (M/F) & Male \\
\hline History of MI & No \\
\hline Previous PTCA & No \\
\hline \multicolumn{2}{|c|}{ Cardiovascular risk factor } \\
\hline Hypertension & Yes \\
\hline Diabetes & No \\
\hline Smoke & Yes \\
\hline Obesity & Moderate \\
\hline \multicolumn{2}{|c|}{ Angiographic data } \\
\hline RCA stenosis & multiple/severe \\
\hline
\end{tabular}




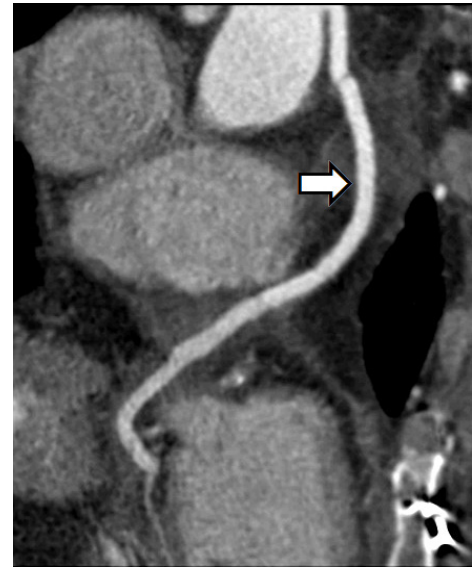

Figure 1: Venous bypass graft of right coronary artery (arrows). Twodimensional curved multiplanar reconstruction.

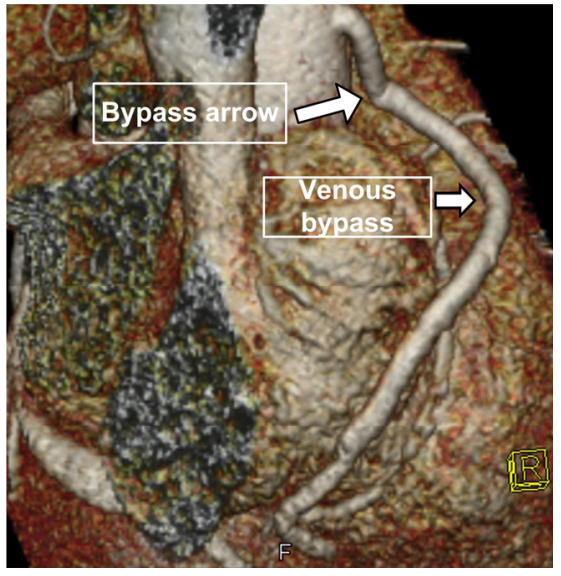

Figure 2: $\quad$ Three-dimensional reconstruction of heart in patient with venous bypass graft (arrows).

Computed tomography has many advantages comparatively to invasive coronary angiography (including a lower complication rate, better ostial imaging and easy visualisation of vessels with anomalous origin). Bypass graft known to be patent at 7 to 10 days after the operation.

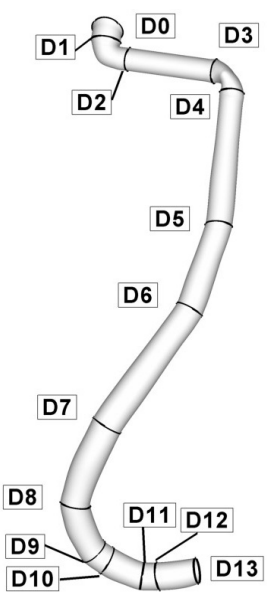

(A)

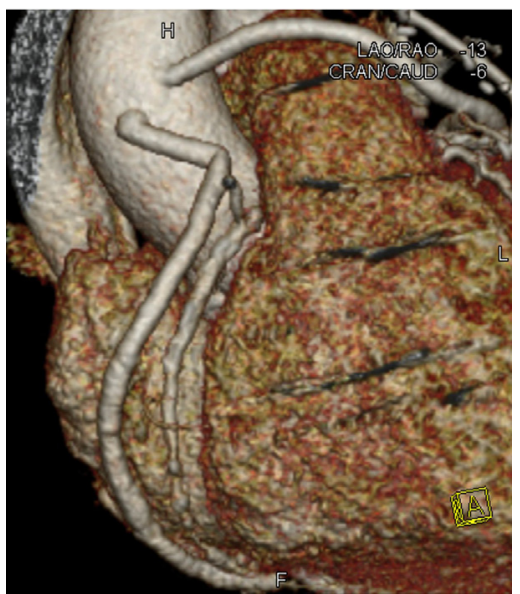

(B)

Figure 3: Venous bypass graft geometry. (A) 3D bypass reconstruction; (B) CT scan images of the bypass. 
Computed tomography methods are used in order to assessing aortocoronary saphenous vein bypass graft patency (Figure 3).

The bypass graft is modelled to be of length $118 \mathrm{~mm}$ with diameter $4.2 \mathrm{~mm}$. In Figure 3, the venous grafts have a uniform circular cross-section, which are larger than the investigated RCA (3.6 mm).

\subsection{Computational fluid dynamics}

Computational assumptions and boundary conditions are the following: the blood is assumed incompressible having dynamic viscosity $(\mu)$ of $0.00408 \mathrm{~Pa}$.s and a density $(\rho)$ of $1050 \mathrm{~kg} / \mathrm{m}^{3}$. The venous bypass walls are considered rigid and impermeable. As usually adopted by most previous investigations, the distensibility of the blood vessel wall is neglected. Torii et al. [9] used the coupled fluid-structure interaction (FSI) method to analyse the human RCA in conjunction with physiological velocity and pressure waveforms in order to investigate the effects of wall compliance on coronary hemodynamics. Comparison of the computational results between the FSI and rigid-wall models showed insignificant difference in TAWSS and OSI. Zeng et al. [10] investigate a disease-free RCA with in vivo flow waveform, physiological realistic compliance data and artery motions.

Johnston et al. [11] compared the effects of different blood viscosity models on the velocity and WSS distributions in the RCA during the cardiac cycle. Their conclude that the Newtonian blood model is a reasonably good approximation for transient blood flow simulation.

The present study, are motivated by the quest to understand the dynamics of flows downstream of narrowed bypass section. In healthy vessels in generally we have a laminar flow, but in the narrowed vessel can lead to relatively highReynolds numbers and disturbed flow. A large number of experimental studies [12-14] and computational [15-17] studies of the hemodynamics in diseased arteries exhibit disordered and turbulent flows in such vessels. Turbulence can significantly affect the pressure and shear stress downstream of the stenosis [18].

Simulations have been carried out over a range of Reynolds numbers (based on the centreline temporally averaged streamwise velocity and the bypass radius) from 215 to 620. The maximum Reynolds number during the cycle was about 600 . In order to predict the flow evolution inside to the narrowed coronary graft, the realizable $\mathrm{k}-\varepsilon$ turbulent model is used in present simulation because this model provides superior performance for flows involving rotation, separation and recirculation.

To solve the time dependent Navier-Stokes equations for an incompressible blood flow the realizable two equation $\mathrm{k}-\varepsilon$ turbulence model is used. The numerical simulation is performed using the commercial CFD FLUENT 6.3 package [19], parallelized across eight $3.2 \mathrm{GHz}$ Intel processors of a TYANPSC T-650 Rx (Tyan Computer Corporation, Taiwan, parallel computing machine with sixteen $3.2 \mathrm{GHz}$ processors). The velocity profile at the inlet section is identical to the real coronary ostium velocity profile [20] (Figure 4). 

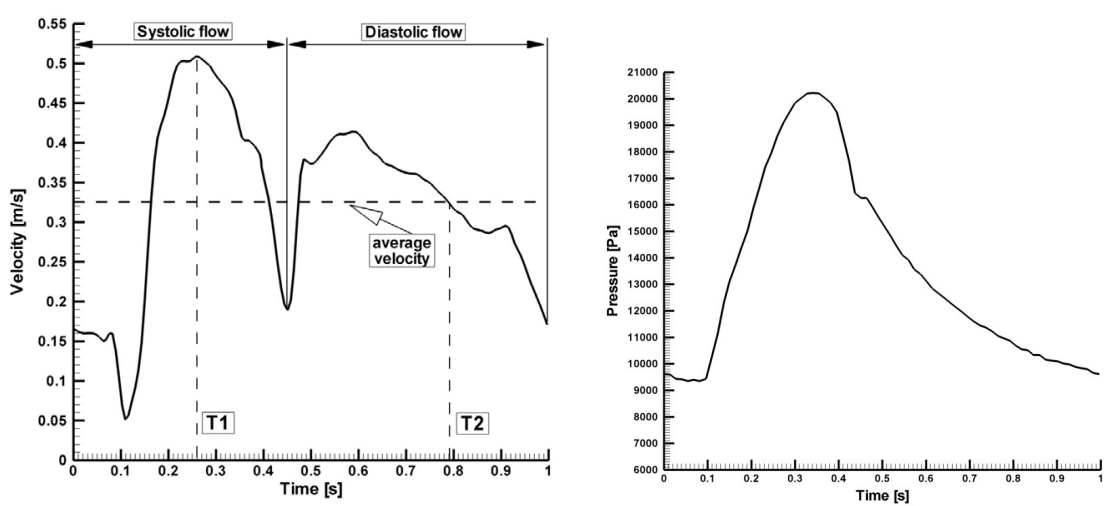

Figure 4: Physiologically realistic boundary conditions, based on flow and pressure waveforms acquired with an intravascular ultrasound Doppler probe in the RCA [20]. Left - velocity input waveform; Right - pressure output waveform.

The venous bypass graft model is reconstructed in GAMBIT [19], using the dimensions provided by the CT measurements. A finer mesh, contained $1,168,765$ elements is used for numerical simulation. Four different meshes with boundary layer mesh were investigated. The number of cells varied between 500,000 and 1,200,000. A high mesh resolution near the walls was needed for accurate values of pressure drop and WSS. Thus the mesh was refined in the near-wall region. A boundary layer consisting of ten rows with a growth factor of 1.15 (ratio between two consecutive layers near the wall) was generated. To model the flow close to the wall, standard wall-function approach was used.

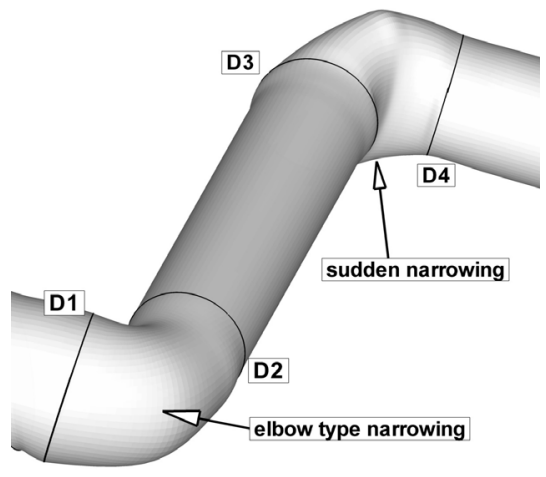

(a)

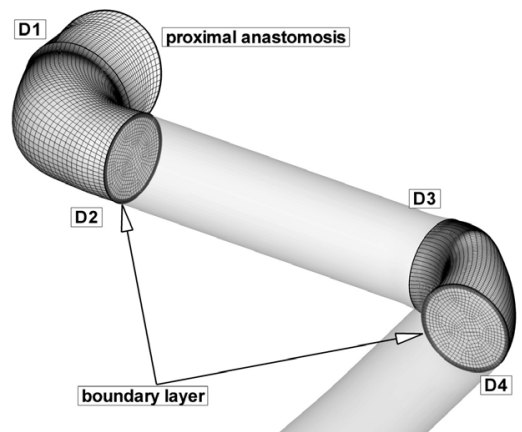

(b)

Figure 5: Detail of the narrowed venous bypass: a) narrowed segment geometry; b) computational domain discretization. 
The governing equations are solved iteratively until convergence of all flow variables is achieved. The convergence criterion was set to 0.001 for the residuals of the continuity equation and of $X, Y$ and $Z$ momentum equations.

Note that recent simulation study of arterial flows presented in the literature $[9,17]$ have shown that about 600,000 grid nodes are required to achieve grid independence in the WSS field. Since our objective is to qualitatively study the unstable flow, the grid containing 1,170,000 cells (Figure 5) can be considered as a good compromise between accuracy and computational cost.

\section{Results}

In the present study the hemodynamic characteristics of the blood flow through the patient-specific bypass model are investigated.

Our results provide data regarding to the hemodynamic parameters for the blood flow in the failed bypass graft under physiological conditions.

At the inlet section we have fully developed flow. In the narrowed region of the graft (between sections $\mathrm{d}_{2}$ and $\mathrm{d}_{4}$ ) a strong region of recirculation is observed (Figure 6). Streamline distribution in narrowed section illustrates also the extension of the recirculation region (Figure 6).

Vortex dynamics prediction is important for estimating the residence times for blood cells. It is relevant because it is accepted that biological processes initiating atherosclerosis are influenced by a combination of fluid and mechanical factors [21].

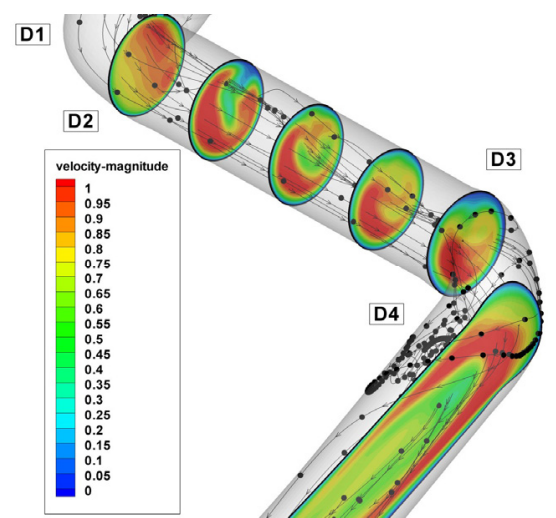

Figure 6: Velocity field in the different cross-section of the venous bypass graft at the time $\mathrm{T} 1=0.26 \mathrm{~s}$ (peak systole).

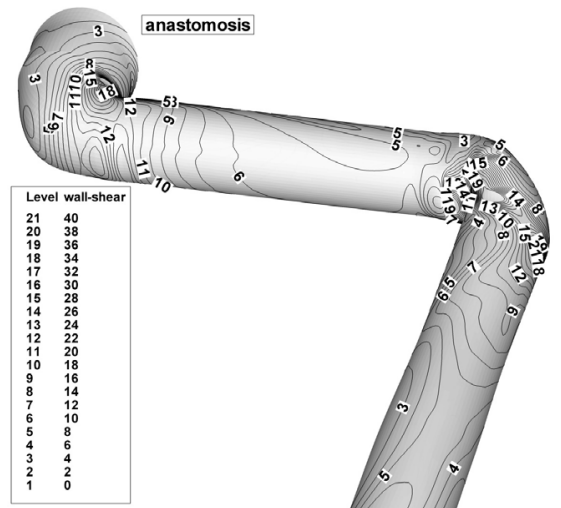

Figure 7: Wall shear stress distribution on the venous bypass graft wall at the time $\mathrm{T} 1=0.26 \mathrm{~s}$.

Figure 7 shows the wall shear stress patterns in both models at the peak systole $(\mathrm{T}=0.26 \mathrm{~s})$. As presented in in Figure 7 the maximum shear stresses occur in the vicinity of the curvature. Presence of the curvature produces acceleration of 
the blood flow and induce distorsion of the flow. Wall shear stress variation during the cardiac cycle is correlated to blood velocity variation.

Pressure distribution and wall shear stress are the relevant parameters from the fluid mechanics point of view. According to the Law of Bernoulli as blood velocity increased the pressure is dropped.

Energy loss is associated with disturbed flow after each curvature. The greater energy loss induces greater pressure drop associated with large re-circulation zones distal to the each curvature and narrowed section (Figures 6 and 7).

Figure 8 present the pressure drop along the narrowed venous bypass graft. Pressure drop coresponding to the different time steps is presented in Figure 9.

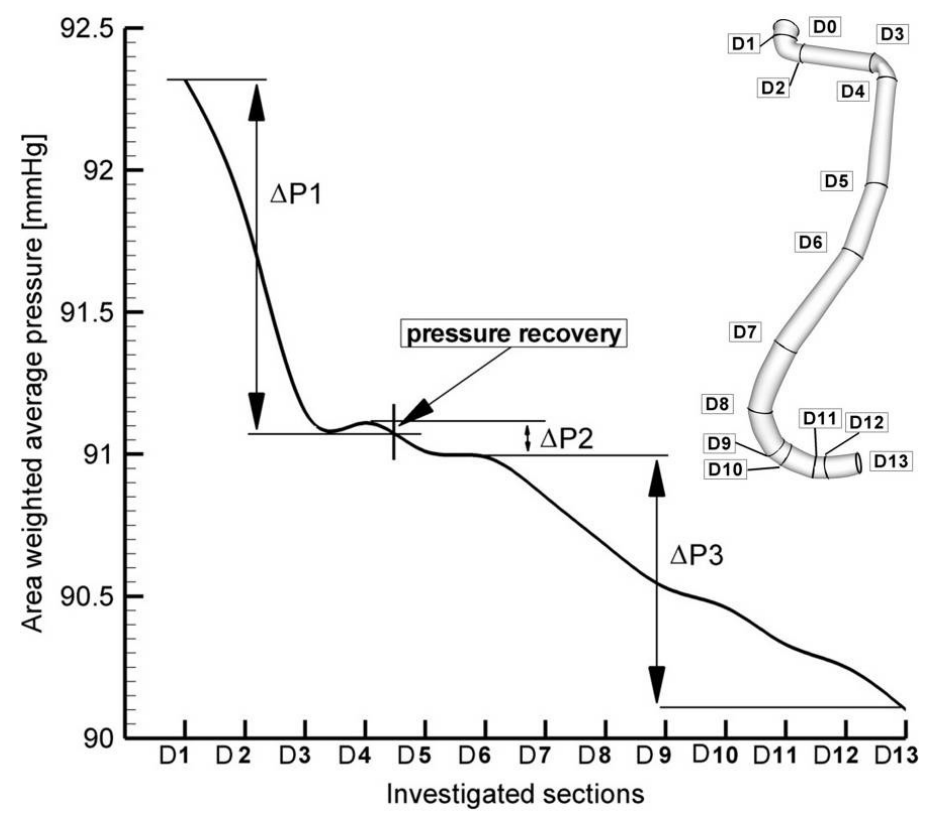

Figure 8: Pressure drop in narrowed venous bypass graft at the time $\mathrm{T} 2=0.79 \mathrm{~s}$. D0 $=$ inlet section (proximal anastomosis), D13 = outlet section (distal end-to-side anastomosis).

Numerical simulation of pulsatile flow through the narrowed bypass graft revealed a three-dimensional instability with profound effects on the flow development. The simulation showed that most of the important flow changes concentrate downstream at the narrowed section. Closer to the constriction, the instability is primarily associated with streamwise flow changes. Blood flow downstream of the constricted bypass region is characterized by a separating region that evolves under the influence of a pulsatile inflow and interacts with the arterial walls in the distal part of the bypass. Vortices and recirculating flow are produced in the vicinity of the constricted section. Vortices evolutions are dependent on the velocity evolution during the cardiac cycle. 


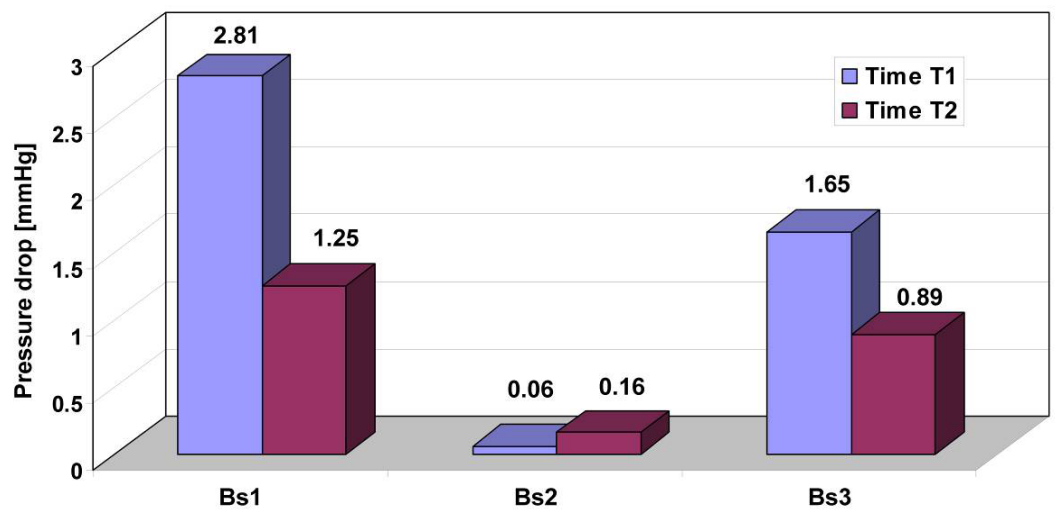

Figure 9: $\quad$ Pressure drop for different time step in the investigated bypass graft. Time T1 and T2 correspond to the time steps indicated in Figure 4. Bs1 = bypass vessel segment between section D0 - D3, Bs2 = bypass vessel segment between section D3 - D6, Bs3 = bypass vessel segment between section D6 - D13 (section position are presented in Figure 3a).

\section{Conclusions}

In the present paper, we investigated the hydrodynamic parameters associated with the blood flow in coronary bypass graft. Coronary bypass flow is complex and cannot be adequately assessed only by interpretation on the coronary angiogram.

The paper has shown that graft geometry is complicated and that the local geometry and velocity distribution in the incoming flow determine the local flow pattern. Velocity and pressure play an important role in any clinical approach to the endothelial dysfunction, and bypass graft heart failure.

In order to create a real physical bypass model, the presented method used commercially available software, both to create an anatomically realistic model and also to reproduce the blood hemodynamics.

Successive curvature and sudden narrowing of the graft induce disturbance of the flow profile, which produce high local WSS and large recirculation regions. Both these conditions create favorable conditions to initiate the graft failure.

The important factor which influences the pressure drop across the bypass graft is the severity of the graft curvature which influences the other hemodynamic indicator namely the extension of the recirculation region.

For physicians, three-dimensional modelling becomes particularly helpful when dealing with complex vascular geometries. The improved understanding of hemodynamic changes produced by such interventions can be applied to determine the best course of the surgical intervention. 


\section{References}

[1] S. Achenbach, Current and future status on cardiac computed tomography imaging for diagnosis and risk stratification, Journal of Nuclear Cardiology, 12 (6), 2005, 703-713.

[2] F. Hornero, V. Cervera, J. Estornell, I. Rodirguez, J.A. Buendia, J.M. Esteban, J.A. Montero, Virtual Vascular Endoscopy for Acute Aortic Dissection, Ann Thorac Surg., 80, 2005, 708-710.

[3] I. Marsahall, S. Zhao, P. Papathanasopoulou, P. Hoskins, X. Yun Xu, MRI and CFD studies of pulsatile flow in healtly and stenosed carotid bifurcation models, Journal of Biomechanics, 37, 2004, 679-687.

[4] S.A. Spicer, C.A. Taylor, Simulation Based Medical Planning for Cardiovascular Disease: Visualization System Foundations, Computer Aided Surgery, 5, 2000, 82-89.

[5] S.I. Bernad, E. Bernad, T. Barbat, V. Albulescu, R. Susan-Resiga, Effects of different types of input waveforms in patient-specific right coronary atherosclerosis hemodynamics analysis. Int. J. of Design \& Nature and Ecodynamics, 5(2), 2010, 1-18.

[6] R. Gardhagen, J. Renner, T. Lanne, M. Karlsson, Subject Specific Wall Shear Stress in the Human Thoracic Aorta, WSEAS TRANSACTIONS on BIOLOGY and BIOMEDICINE, 10 (3), 2006, 609-614.

[7] C.N. Yung, K.J. De Witt, S. Subramanian, A.A. Afjeh, T.G. Keith, Threedimensional pulsatile flow through a bifurcation, International Journal of Numerical Methods for Heat \& Fluid Flow, 7 (8), 1997, 843-862.

[8] M.J. Marques, J. Koen, H.J. Spruijt, C. Boer, N. Westerhof, C.A. Visser, F.C. Visser, The diastolic flow-pressure gradient relation in coronary stenoses in humans, J. Am. Coll. Cardiol., 39, 2002, 1630-1636.

[9] R. Torii, N.B. Wood, N. Hadjiloizou, A.W. Dowsey, A.R. Wright, A.D. Hughes, J. Davies, D.P. Francis, J. Mayet, G-Z. Yang, S.A.McG. Thom, X.X. Yun, Fluid-structure interaction analysis of a patient-specific right coronary artery with physiological velocity and pressure waveforms. Communications in Numerical Methods in Engineering, 25, 2009, 565-580.

[10] D. Zeng, E. Boutsianis, M Ammann, K. Boomsma, S. Wildermuth, D Poulikakos, A study on the Compliance of a Right Coronary Artery and Its Impact on Wall Shear Stress, Journal of Biomechanical Engineering, 130, 2008, 041014-1:11.

[11] B.M. Johnston, P.R. Johnston, S. Corney, D. Kilpatrick, Non-Newtonian blood flow in human right coronary arteries: transient simulations. Journal of Biomechanics, 39, 2005, 1116-1128.

[12] C. Clark, The propagation of turbulence produced by a stenosis, $J$. Biomech., 113, 1980, 591-604.

[13] A.M.A. Khalifa, D.P. Giddens, Characterization and evolution of poststenotic flow disturbances, J. Biomech., 14, 1981, 279-296.

[14] B.M. Kim, W.H. Corcoran, Experimental measurements of turbulence spectra distal to stenoses, J. Biomech., 7, 1974 335-342. 
[15] F. Ghalichi, X. Deng, Turbulence detection in a stenosed artery bifurcation by numerical simulation of pulsatile blood flow using the low-Reynolds number turbulence model, Biorheology, 40, 2003, 637-654.

[16] R. Mittal, S.P. Simmons, H.S. Udaykumar, Application of large-eddy simulation to the study of pulsatile flow in a modelled arterial stenosis, $J$. Biomech. Engrg. 123, 2001, 325-332.

[17] V.T. Rayz, S. A. Berger, D. Salomer, Transitional flow in arterial fluid dynamics, Comput. Methods Appl. Mech. Engrg., 196, 2007, 3043-3048.

[18] H.M. Loree, R.D. Kamm, C.M. Atkinson, R.T. Lee, Turbulent pressure fluctuations on surface of model vascular stenoses, Am. J. Physiol., 261, 1991, H644-H650.

[19] FLUENT 6.3 User's Guide, Fluent Incorporated, 2006.

[20] R. Torii, N.B. Wood, N. Hadjiloizou, A.W. Dowsey, A.R. Wright, A.D. Hughes, J. Davies, D.P. Francis, J. Mayet, G-Z Yang, S.A. Thom, \& X.X. Yun, Differences in coronary artery haemodynamics due to changes in flow and vascular geometry after percutaneous coronary intervention. Heart, 94, 2008, A1-A4.

[21] J.A. Moore, D.A. Steiman, D.W. Holdsworth, C.R. Ethier, Accuracy of computational hemodynamics in complex arterial geometries reconstructed from magnetic resonance imaging. Ann. Biomed. Eng., 27, 1999, 32-41. 